\title{
Blood flow through axially symmetric sections of compliant vessels: new effective closed models
}

\author{
S. Čanić ${ }^{1}$, J. Tambača ${ }^{2}$, A. Mikelić ${ }^{3}$, C.J. Hartley ${ }^{4}$, D. Mirković ${ }^{5}$, J. Chavez ${ }^{6}$, D. Rosenstrauch ${ }^{7}$ \\ ${ }^{1}$ Department of Mathematics, University of Houston, Houston, TX 77204, USA \\ ${ }^{2}$ Department of Mathematics, University of Zagreb, Bijenička 30, Croatia \\ ${ }^{3}$ Université Claude Bernard Lyon 1, 69622 Villeurbanne Cedex, France \\ ${ }^{4}$ Sections of Cardiovascular Sciences, Dept. of Medicine, Baylor College of Medicine, Houston, TX 77030 \\ ${ }^{5}$ Department of Computer Science, University of Houston, TX 77204 \\ ${ }^{6}$ Department of Physics, University of Houston, TX 77204 \\ ${ }^{7}$ Department of Internal Medicine/Cardiology, UTHSC, TX 77030
}

\begin{abstract}
Due to a tremendous complexity of the human cardiovascular system it remains unfeasible to numerically simulate larger sections of the circulatory system using the full three-dimensional (viscous, incompressible Navier-Stokes) equations for blood flow in compliant vessels. Several "effective" one-dimensional models have been used to simplify the calculation in the axially symmetric sections. All of the onedimensional models assume an ad hoc axial velocity profile to obtain a closed system of equations, and the Law of Laplace (the independent ring model) to model the vessel wall behavior. In this work we obtain an effective system of equations with the following two novel features: (1) the effective equations do not require an ad hoc closure assumption (the closure follows from the analysis of the original three-dimensional equations) and (2) the vessel wall is modeled as a nonlinearly elastic shell using the Koiter model or the nonlinear membrane model. The first novelty provides a higher-order accurate solution to the original three-dimensional problem, and the second allows deformations of the vessel wall that are not necessarily small.

An efficient, fast ("real-time") numerical algorithm based on the coupled finite difference-finite element method has been obtained. Our numerical solutions show secondary flows in certain geometries that cannot be captured with onedimensional models.
\end{abstract}

\section{INTRODUCTION}

We study the flow of blood in medium-to-large size arteries. We are interested in a time-dependent flow through the axially symmetric sections of the vascular system. The Navier-Stokes equations for the unsteady axially symmetric flow of a Newtonian incompressible fluid have been shown to be a good model for the problem, see e.g., [7]. Written in cylindrical coordinates (in the Eulerian framework) the equations, defined on a cylindrical domain $\Omega(t)=\{(r, z) \mid 0<$ $r<R(z, t), 0<z<L\}$, are given by

$$
\begin{aligned}
& \rho\left\{\frac{\partial v_{r}}{\partial t}+\vec{v} \cdot \nabla_{r, z} v_{r}\right\}+\frac{\partial p}{\partial r}=\mu\left\{\Delta_{r, z} v_{r}-\frac{1}{r^{2}} v_{r}\right\}, \\
& \rho\left\{\frac{\partial v_{z}}{\partial t}+\vec{v} \cdot \nabla_{r, z} v_{z}\right\}+\frac{\partial p}{\partial z}=\mu \Delta_{r, z} v_{z},
\end{aligned}
$$

\footnotetext{
${ }^{1}$ Research of the author is partly supported by the National Science Foundation under Grants DMS-9977372, DMS-0245513, and by the Texas Higher Education Board ARP-Mathematics 003652-0112-2001.

${ }^{4}$ Research of the author is partly supported by the National Institutes of Health under Grant HL22512

${ }^{5}$ Research of the author is partly supported by the National Science Foundation under REU Grant Supplement to DMS-9977372
}

$$
\frac{\partial v_{r}}{\partial r}+\frac{\partial v_{z}}{\partial z}+\frac{v_{r}}{r}=0
$$

where $\vec{v}=\left(v_{r}, v_{z}\right)$ is fluid velocity, $p$ is the pressure, $\rho$ is fluid density and $\mu$ the viscosity coefficient. The differential operators $\Delta_{r, z}$ and $\vec{v} \cdot \nabla_{r, z}$ are defined by the following

$$
\Delta_{r, z}=\frac{\partial^{2}}{\partial r^{2}}+\frac{\partial^{2}}{\partial z^{2}}+\frac{1}{r} \frac{\partial}{\partial r}, \vec{v} \cdot \nabla_{r, z}=v_{r} \frac{\partial}{\partial r}+v_{z} \frac{\partial}{\partial z} \text {. }
$$

We will be assuming that the vessel walls are compliant utilizing two nonlinear elastic shell models to study their behavior: one is the nonlinear membrane model, see also [6], and the other is the nonlinear Koiter shell model [5], both applied for the cylindrical shell with thickness $h>0$. In contrast with the first model, the Koiter model accounts for the bending rigidity, neglected in the nonlinear membrane model due to the intrinsic assumption of infinitesimally small thickness. In the "unstressed", reference state the cylinder is $L>0$ units long and $2 R>0$ units wide. Throughout the paper we will be assuming that the aspect ratio $\epsilon=R / L>0$ is small and that $h / R$ is of order $O(1)$ for the nonlinear Koiter model. Furthermore, we will be assuming that the lateral wall $\Sigma(t)$ of the cylinder $\Omega(t)$ allows only radial displacements $\eta(z, t)$ so that $R(z, t)=R+\eta(z, t)$. The motion of the vessel wall, given in Lagrangian coordinates, is governed by the radial component of the contact force, which is in the case of the nonlinearly elastic membrane given by

$$
\begin{aligned}
F_{r}^{\mathrm{M}}= & \rho_{w} h \frac{\partial^{2} \eta}{\partial t^{2}}-\frac{h}{2}\left\{\frac { \partial } { \partial z } \left[\left(\frac{E}{1-\nu^{2}}\left(\frac{\partial \eta}{\partial z}\right)^{2}\right.\right.\right. \\
+ & \left.\left.\frac{E \nu}{1-\nu^{2}}\left(\frac{2}{R} \eta+\frac{1}{R^{2}} \eta^{2}\right)\right) \frac{\partial \eta}{\partial z}\right]+\left(\frac{1}{R}+\frac{1}{R^{2}} \eta\right) \\
& \left.\left(\frac{E}{1-\nu^{2}}\left(\frac{2}{R} \eta+\frac{1}{R^{2}} \eta^{2}\right)+\frac{E \nu}{1-\nu^{2}}\left(\frac{\partial \eta}{\partial z}\right)^{2}\right)\right\}
\end{aligned}
$$

where $\rho_{w}$ is the wall volumetric mass, $E$ is the Young's modulus and $0<\nu \leq 0.5$ is the Poisson ratio. The contact force for the nonlinear Koiter model can be found in [1], [5]. Only the leading order contribution, given in (5), is relevant in the derivation of the models.

The fluid equations are coupled with the vessel wall equation through the deformed interface $R+\eta(z, t)$ by 
requiring continuity of velocity and continuity (balance) of forces. The continuity of velocity reads

$$
v_{r}(R+\eta, z, t)=\frac{\partial \eta}{\partial t}(z, t), \quad v_{z}(R+\eta, z, t)=0 .
$$

Since the fluid contact force is typically given in Eulerian coordinates and the structure (vessel wall) contact force is in Lagrangian coordinates to perform the coupling we need the Jacobian of the transformation $J=\left(1+(\partial \eta / \partial z)^{2}\right)^{1 / 2}$ so that the continuity of contact forces reads

$$
-F_{r}=\left(\left(p-p_{r e f}\right) I-2 \mu D(v)\right) \vec{n} \cdot \vec{e}_{r}\left(1+\frac{\eta}{R}\right) J
$$

where $D(v)=\frac{1}{2}\left(\nabla v+(\nabla v)^{t}\right)$ is the rate of strain tensor and $F_{r}$ is the radial component of the contact force associated with the structure, e.g., $F_{r}=F_{r}^{M}$.

Initially, the cylinder is filled with fluid and the entire structure is at rest: $\eta=\frac{\partial \eta}{\partial t}=0$ and $v=0$ at $t=0$.

A time-dependent pressure head data at the inlet and at the outlet boundary drive the problem:

$$
\begin{array}{ll}
p+\rho v_{z}^{2} / 2=P_{0}(t)+p_{\text {ref }} v_{r}=0, \eta=0 & \text { for } z=0 \\
p+\rho v_{z}^{2} / 2=P_{L}(t)+p_{\text {ref }} v_{r}=0, \eta=0 & \text { for } z=L
\end{array}
$$

\section{Methods}

Our goal is to obtain a set of closed reduced (simplified) equations approximating this axially symmetric threedimensional problem to the order $\epsilon^{2}$. For this purpose we derive a weak formulation of the problem, obtain the energy estimates and use them to derive the a priori solution estimates. They determine the upper bounds for the unknown functions $\vec{v}, \eta$ in terms of the parameters in the problem. See [1]. The a priori bounds determine the characteristic scales $V$ and $\Xi$ in $\vec{v}=V \bar{v}, \eta=\Xi \bar{\eta}, p=\rho V^{2} \bar{p}$ and we can perform the nondimensional analysis to obtain a system of two-dimensional reduced equations that solve the original problem to the $\epsilon^{2}$ order:

$$
\begin{aligned}
S h\left(\frac{\partial \bar{v}_{z}}{\partial \bar{t}}+\bar{v} \cdot \nabla \bar{v}_{z}\right)+\frac{\partial \bar{p}}{\partial \bar{z}} & =\frac{1}{R e} \frac{1}{\bar{r}} \frac{\partial}{\partial \bar{r}}\left(\bar{r} \frac{\partial \bar{v}_{z}}{\partial \bar{r}}\right) \\
\frac{\partial \bar{p}}{\partial \bar{r}} & =0, \\
\frac{1}{\bar{r}} \frac{\partial}{\partial \bar{r}}\left(\overline{r v}_{\bar{r}}\right)+\frac{\partial \bar{v} \bar{z}}{\partial \bar{z}} & =0,
\end{aligned}
$$

where $R e=\frac{\rho V R^{2}}{\mu L}$ and $S h=\frac{L \omega}{V}$, and the characteristic frequency $\omega=$ cons. $((h E) /(\rho R(1+\nu)))^{1 / 2} / L$. The leading order behavior for the radial contact force for the nonlinear membrane model, denoted by $\bar{F}_{r}^{M}$, and for the nonlinear Koiter model, denoted by $\bar{F}_{r}^{K}$, are given by

$$
\begin{gathered}
\bar{F}_{r}^{M}=\frac{1}{2} \frac{\mathcal{P} \bar{\eta}}{1-\nu}\left(2+\frac{\Xi}{R} \bar{\eta}\right)\left(1+\frac{\Xi}{R} \bar{\eta}\right) \\
\bar{F}_{r}^{K}=\bar{F}_{r}^{M}+\frac{1}{12}\left(\frac{h}{R}\right)^{2} \frac{\mathcal{P} \bar{\eta}}{1-\nu} \\
\mathcal{P}^{2}=\max _{t, z} \hat{p}^{2}+T^{2}\left\{\frac{4}{T} \int_{0}^{T} \max _{z} \partial_{t} \hat{p}^{2} d \tau+\frac{1}{T} \int_{0}^{T}|A|^{2} d \tau\right\} \\
\text { where } \hat{p}(z, t)=\frac{A(t)}{L} z+P_{0}(t) \text { and } A(t)=P_{L}(t)-P_{0}(t) .
\end{gathered}
$$

This reduced two-dimensional problem is difficult to solve. For this reason we continue the analysis in an attempt to obtain a set of reduced, effective equations that can be easily solved numerically. A typical approach at this point is to average equations (1), (3) across the vessel cross-section to obtain a one-dimensional system. See [2], [10]. Since the underlying problem is nonlinear, this procedure requires additional information such as the axial velocity profile to obtain a closed system. There are several choices typically used in the literature [8], [2], [9]. They range from an almost flat velocity profile to account for the non-Newtonian nature of blood flow, to the Poisseuile velocity profile approximating Newtownian fluid. In our present paper we continue in a different direction. We further simplify equations (1)(3) without assuming an ad hoc closure. Using the ideas borrowed from the homogenization theory of flows in porous media, see [3], we have derived a closed system of reduced equations. It was proved in [1] that they solve the original problem to the $\epsilon^{2}$ order. We list the basic assumptions and the resulting system in dimensional form below.

Assumptions. The following assumptions have been used in the derivation of the reduced equations below:

- The domain is axially symmetric.

- Aspect ratio is small $\epsilon=R / L<<1$.

- Ratio $h / L<<1$ to use the cylindrical shell equations.

- Ratio $h / R=O(1)$ ( $h$ and $R$ are of the same order of magnitude) for the Koiter model.

- The longitudinal displacement is negligible.

- The radial displacement is not too big: $\Xi / R \leq \epsilon$.

- The $z$-derivatives of all the non-dimensional quantities are of order 1. They are not necessarily small.

- The Strouhal number $S h$ is not small: $S h>1$ (This is the flow regime e.g. in the iliac arteries.)

The assumption on the size of the radial displacement $\Xi / R \leq \epsilon$ is typically satisfied for human arteries [4] since, e.g., for the values listed in Table I this reads that the maximum radial displacement is about $7 \%$ of the nonstressed radius $R$. We remark that using linear models with this displacement size would produce different results from the ones presented in this paper. The model equations presented in this manuscript capture the "intermediate" behavior between linearized elasticity assuming rather small deformations and strongly nonlinear behavior with large deformations that are not typically observed in the human vascular system.

Reduced Equations. We expand the unknown functions with respect to $\epsilon$ and $\delta=\Xi / R$ and look for a solution which is an $\epsilon^{2}$-approximation of the original equations. The approximate solution has the following form

$$
\vec{v}=\left(v_{r}, v_{z}\right)=\left(v_{r}^{1,0}, v_{z}^{0,0}+v_{z}^{0,1}+v_{z}^{1,0}\right), \eta=\eta^{0,0}+\eta^{0,1}
$$

where the first superscript corresponds to the expansion with respect to $\epsilon$ and the second with respect to $\delta$.

Denote

$$
C=\frac{h E}{R^{2}\left(1-\nu^{2}\right)}\left(1+\frac{\alpha^{2}}{12}\right), \alpha=\left\{\begin{array}{ll}
0, & \text { membrane } \\
\frac{h}{R}, & \text { Koiter }
\end{array}\right. \text {. }
$$


The model equations are the following.

Approximation 0, 0

$$
\begin{aligned}
\frac{\partial \eta^{0,0}}{\partial t}+\frac{1}{R} \frac{\partial}{\partial z} \int_{0}^{R} r v_{z}^{0,0} d r & =0 \\
\rho \frac{\partial v_{z}^{0,0}}{\partial t}-\mu \frac{1}{r} \frac{\partial}{\partial r}\left(r \frac{\partial v_{z}^{0,0}}{\partial r}\right) & =-\frac{\partial p^{0,0}}{\partial z} .
\end{aligned}
$$

The boundary and initial conditions are

$$
\begin{aligned}
& \left.v_{z}^{0,0}\right|_{r=0}-\text { bounded, }\left.\quad v_{z}^{0,0}\right|_{r=R}=0,\left.\quad v_{z}^{0,0}\right|_{t=0}=0, \\
& \left.\eta^{0,0}\right|_{t=0}=0,\left.\quad \eta^{0,0}\right|_{z=0}=\frac{P_{0}(t)}{C},\left.\quad \eta^{0,0}\right|_{z=L}=\frac{P_{L}(t)}{C},
\end{aligned}
$$

with the 0,0 -pressure displacement relation

$$
p^{0,0}=C \eta^{0,0} \text {. }
$$

Approximation 0,1

$$
\begin{aligned}
& \frac{\partial}{\partial t} \eta^{0,1}+\frac{1}{R} \frac{\partial}{\partial z} \int_{0}^{R} r v_{z}^{0,1} d r=-\frac{1}{2 R} \frac{\partial}{\partial t}\left(\eta^{0,0}\right)^{2} \\
& \rho \frac{\partial v_{z}^{0,1}}{\partial t}-\mu \frac{1}{r} \frac{\partial}{\partial r}\left(r \frac{\partial v_{z}^{0,1}}{\partial r}\right)=-\frac{\partial p^{0,1}}{\partial z}
\end{aligned}
$$

The boundary and initial conditions are:

$$
\begin{gathered}
\left.v_{z}^{0,1}\right|_{r=0} \text { bound., }\left.v_{z}^{0,1}\right|_{r=R}=-\left.\eta^{0,0} \frac{\partial v_{z}^{0,0}}{\partial r}\right|_{r=R},\left.v_{z}^{0,1}\right|_{t=0}=0, \\
\left.\eta^{0,1}\right|_{t=0}=0,\left.\quad \eta^{0,1}\right|_{z=0}=0,\left.\quad \eta^{0,1}\right|_{z=L}=0
\end{gathered}
$$

with the 0,1-pressure displacement relation

$$
p^{0,1}=C\left(\eta^{0,1}+\frac{1}{2 R} \frac{1-\alpha^{2} / 6}{1+\alpha^{2} / 12}\left(\eta^{0,0}\right)^{2}\right) .
$$

Approximation 1,0

$$
v_{r}^{1,0}(r, z, t)=-\frac{1}{r} \int_{0}^{r} \xi \frac{\partial v_{z}^{0,0}}{\partial z}(\xi, z, t) d \xi,
$$

$\rho \frac{\partial v_{z}^{1,0}}{\partial t}-\frac{\mu}{r} \frac{\partial}{\partial r}\left(r \frac{\partial v_{z}^{1,0}}{\partial r}\right)=-\rho v_{r}^{1,0} \frac{\partial v_{z}^{0,0}}{\partial r}-\rho v_{z}^{0,0} \frac{\partial v_{z}^{0,0}}{\partial z}$.

with the initial and boundary conditions

$$
\left.v_{z}^{1,0}\right|_{r=0}-\text { bounded, }\left.\quad v_{z}^{1,0}\right|_{r=R}=0,\left.\quad v_{z}^{1,0}\right|_{t=0}=0 .
$$

Numerical Solver. Note that the approximation 1,0 can easily be calculated once the approximation 0,0 has been obtained. Furthermore, the 0,0 and 0,1 approximations are of the same form. We simplify their calculation by introducing the following auxiliary problem: let us denote by $\zeta$ the unique solution of the problem

$$
\begin{aligned}
& \varrho_{F} \frac{\partial \zeta}{\partial t}-\mu_{F} \frac{1}{r} \frac{\partial}{\partial r}\left(r \frac{\partial \zeta}{\partial t}\right)=0, \\
& \left.\zeta\right|_{t=0}-\text { bounded, }\left.\quad \zeta\right|_{r=R}=0,\left.\quad \zeta\right|_{t=0}=1
\end{aligned}
$$

and denote

$$
\mathcal{K}(t)=2 \int_{0}^{R} r \zeta(r, t) d r .
$$

Then the solution of the problem

$$
\begin{aligned}
& \varrho_{F} \frac{\partial w}{\partial t}-\mu_{F} \frac{1}{r} \frac{\partial}{\partial r}\left(r \frac{\partial w}{\partial t}\right)=f, \\
& \left.w\right|_{t=0}-\text { bounded, }\left.\quad w\right|_{r=R}=0,\left.\quad w\right|_{t=0}=0,
\end{aligned}
$$

where $f$ is a function of $z$ and $t$ only, is given by

$w(r, z, t)=\frac{1}{\varrho_{F}}(\zeta * f)(r, z, t)=\frac{1}{\varrho_{F}} \int_{0}^{t} \zeta(r, t-\tau) f(z, \tau) d \tau$.

We use this solution operator to "solve" for the velocity and plug this into the equations for the displacements $\eta^{0,0}$ and $\eta^{0,1}$ obtaining:

$$
\begin{aligned}
& \frac{\partial \eta^{0,0}}{\partial t}=\frac{C}{2 R \varrho_{F}} \mathcal{K} * \frac{\partial^{2} \eta^{0,0}}{\partial z^{2}}, \\
& \left.\eta^{0,0}\right|_{t=0}=0,\left.\eta^{0,0}\right|_{z=0}=\frac{P_{0}(t)}{C},\left.\eta^{0,0}\right|_{z=L}=\frac{P_{L}(t)}{C},
\end{aligned}
$$

and

$$
\begin{aligned}
& \frac{\partial \eta^{0,1}}{\partial t}=\frac{C}{2 \varrho_{F} R} \mathcal{K} * \frac{\partial^{2} \eta^{0,1}}{\partial z^{2}}+S^{0,1}, \\
& \left.\eta^{0,1}\right|_{t=0}=0,\left.\quad \eta^{0,1}\right|_{z=0}=0,\left.\quad \eta^{0,1}\right|_{z=L}=0,
\end{aligned}
$$

where the source term is given by

$$
\begin{array}{r}
S^{0,1}=\frac{1}{2 R}\left(-\frac{\partial}{\partial t}\left(\eta^{0,0}\right)^{2}+R^{2} \frac{\partial}{\partial z}\left(\left.\eta^{0,0} \frac{\partial v_{z}^{0,0}}{\partial r}\right|_{r=R}\right)\right. \\
\left.+D \frac{\partial}{\partial z}\left(\mathcal{K} *\left(\eta^{0,0}\right)^{2}\right)-\frac{\partial}{\partial z}\left(\mathcal{K} * \frac{\partial}{\partial t}\left(\left.\eta^{0,0} \frac{\partial v_{z}^{0,0}}{\partial r}\right|_{r=R}\right)\right)\right)
\end{array}
$$

with $D=\frac{C}{2 \rho R}\left(1-\alpha^{2} / 6\right) /\left(1+\alpha^{2} / 12\right)$. We summarize the entire numerical procedure below. The numerical algorithm is a combination of the finite difference method (FDM) used to solve for wave propagation in the elastic structure and a finite element method (FEM) used to solve for the velocity of the fluid.

\section{THE NUMERICAL SCHEME}

(i) Solve the auxiliary problem (12) using 1D FEM

(ii) Compute $\mathcal{K}$ using numerical integration

(iii) Approximation 0,0

a) solve (13) for $\eta^{0,0}$ using implicit FDM

b) solve (7) for $v_{z}^{0,0}$ using 1D FEM

(iv) Approximation 0,1

a) solve (14) for $\eta^{0,1}$ using implicit FDM

b) solve (9) for $v_{z}^{0,1}$ using 1D FEM

(v) Approximation 1,0

a) solve (10) for $v_{r}^{1,0}$ using numerical integration

b) solve (11) for $v_{z}^{1,0}$ using 1D FEM

(vi) Compute the total approximation

$$
v_{r}=v_{r}^{1,0}, v_{z}=v_{z}^{0,0}+v_{z}^{0,1}+v_{z}^{1,0}, \eta=\eta^{0,0}+\eta^{0,1} .
$$

First note that the problems for $\zeta, v_{z}^{0,0}, v_{z}^{0,1}$ and $v_{z}^{1,0}$ are all of the same form so the mass and stiffness matrices in the FEM method are equal, up to the boundary condition. Therefore they need to be generated only once. The same argument holds for the FDM used to solve for $\eta^{0,0}$ and $\eta^{0,1}$. The main difficulty in the entire calculation is in calculating the time integral appearing in the problems for $\eta^{0,0}$ and $\eta^{0,1}$.

Notice that our algorithm consists of solving a sequence of 1D problems, so its complexity is the same that of solving a $1 \mathrm{D}$ problem.

Numerical Simulations. We present the numerical simulations for the data, shown in Table I, corresponding to iliac 


\begin{tabular}{|l|l|}
\hline \hline PARAMETERS & VALUES \\
\hline Characteristic radius: $R$ & $0.0025 \mathrm{~m}$ \\
\hline Characteristic length $: L$ & $0.065 \mathrm{~m}$ \\
\hline Dynamic viscosity: $\mu$ & $3.5 \times 10^{-3} \mathrm{~kg} / \mathrm{m} / \mathrm{s}$ \\
\hline Young's modulus: $\mathrm{E}$ & $10^{5}-8 \times 10^{5} \mathrm{~Pa}, E=10^{5} \mathrm{~Pa}$ used \\
\hline Poisson ratio $\nu$ & 0.5 \\
\hline Wall thickness: $\mathrm{h}$ & $0.002 \mathrm{~m}$ \\
\hline Blood density: $\rho$ & $1050 \mathrm{~kg} / \mathrm{m}^{3}$ \\
\hline
\end{tabular}

TABLE I

PARAMETER VALUES

arteries. The corresponding Strouhal number is $S h=14.25$ and the local Reynolds number $R e_{0}=\frac{2 R V \rho}{\mu}=750$. Figure 1 shows a small recirculation zone occurring at a fraction of a second at which the geometry of the vessel admits this kind of secondary flow. The red dot on the graph of the inlet pressure shows the position in the cardiac cycle when the snap-shot is taken. The corresponding axial
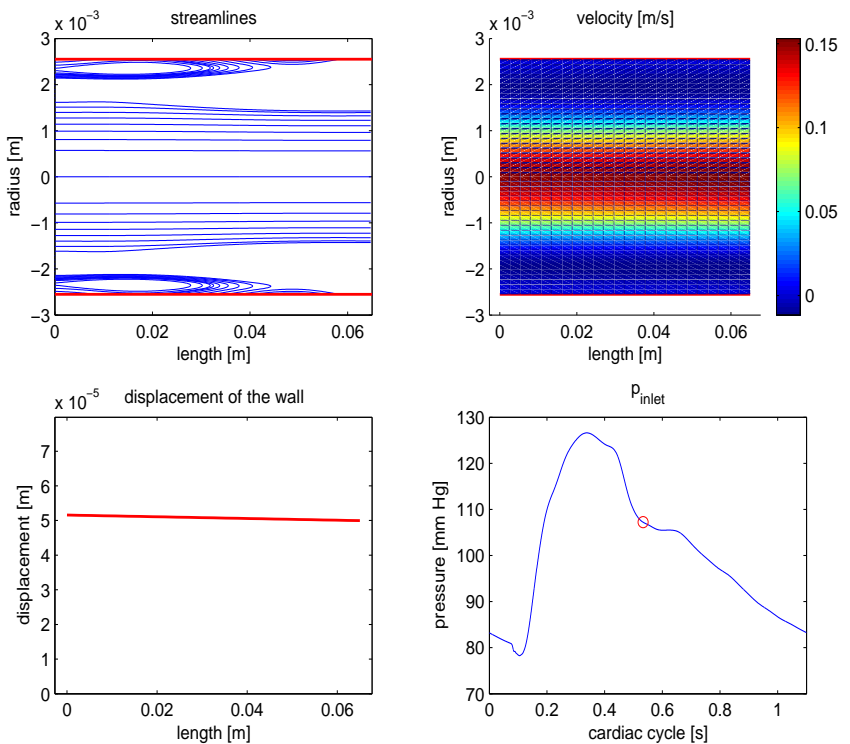

Fig. 1. Flow in a compliant vessel of radius $R=2.5 \mathrm{~mm}$, with Young's modulus $E=10^{5} \mathrm{~Pa}$.

velocity profiles in the middle of the vessel, taken at six different times in the cardiac cycle are shown in Figure 2. As expected, the velocity profiles are similar to the Womersley flow profiles [11]. This shows that using the Poisseuille flow (quadratic polynomial in the radial variable) as an ad hoc closure assumption in one-dimensional models will not produce an $\epsilon^{2}$ approximation of the flow of a Newtonian fluid in elastic tubes.

\section{DISCUSSION}

Our numerical simulations show that the system of equations presented in this paper captures various phenomena in the flow of a Newtonian viscous fluid through a nonlinearly elastic tube that cannot be captured using the simplified one-dimensional models typically used in the simulation of blood flow through axially symmetric sections of the

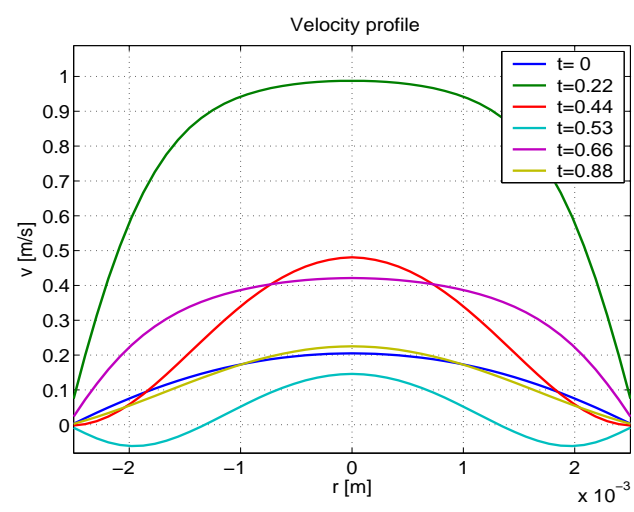

Fig. 2. Axial velocity profiles at 6 time-positions in the cardiac cycle.

vascular system. The numerical algorithm complexity is that of a 1D algorithm, taking typically several minutes on a PC for a calculation of flow in several cardiac cycles. Our model provides an approximation of the original three-dimensional problem with higher accuracy. More precisely, it has been mathematically proven that the model is an $\epsilon^{2}$-approximation to the original equations.

\section{REFERENCES}

[1] S. Čanić, J. Tambača and A. Mikelić. Coupling between the flow of a viscous incompressible fluid and the nolinearly elastic cylindrical shell of Koiter type: the effective equations and a numerical solver In preparation.

[2] S. Canić, and E. H. Kim. Mathematical Analysis of the Quasilinear Effects in a Hyperbolic Model of Blood Flow through Compliant Axisymmetric Vessels. Mathematical Methods in Applied Sciences, 26 (14) (2003), 1161-1186.

[3] S. Canić, D. Lamponi, A. Mikelić and J. Tambača. Self-Consistent Effective Equations Modeling Blood Flow in Medium-to-Large Compliant Arteries. submitted to SIAM J. Multiscale Analysis and Simulation (2003)

[4] Y.C. Fung. Biomechanics: Circulation. Springer New York 1993. Second Edition.

[5] Koiter, W. T. On the nonlinear theory of thin elastic shells. I, II, III. Nederl. Akad. Wetensch. Proc. Ser. B, 69: 1-17, 18-32, 33-54, 1966.

[6] X. Ma, G.C. Lee and S.G. Lu Numerical Simulation for the ropagation of Nonlinear pulsatile Waves in Arteries. ASME Journal o Biomechanical Engineering 114:490-496, 1992.

[7] McDonald's Blood Flow in Arteries: Theoretical, experimental and clinical principles, Fourth Edition Wilmer W. Nichols and Michael F. O'Rourke, Arnold and Oxford University Press Inc. New York, London, Sydney, Auckland.

[8] M.S. Olufsen, C.S. Peskin, W.Y. Kim, E.M. Pedersen, A. Nadim, J. Larsen, Numerical Simulation and Experimental Validation of Blood Flow in Arteries with Structured-Tree Outflow Conditions, Annals of Biomedical Engineering 28 (2000), 1281-1299.

[9] N. P. Smith, A. J. Pullan, P. J. Hunter. An Anatomically Based Model of Transient Coronary Blood Flow in the Heart. SIAM J. Appl. Math. 62(3) (2002), 990-1018.

[10] A. Quarteroni, M. Tuveri and A. Veneziani. Computational vascular fluid dynamics: problems, models and methods. Survey article, Comput. Visual. Sci. 2 (2000), 163-197.

[11] J.R. Womersley. Method for the calculation of velocity, rate of flow and viscous drag in arteries when the pressure gradient is known $\mathrm{J}$. Physiol. 127 (1955), 553-563. 